\title{
La duración de los tratamientos psicológicos: diferencias entre casos de corta, media y larga duración*
}

\author{
Duration of Psychological Treatments: Differences \\ among Cases of Short, Medium and Long Term
}

Recibido: octubre 24 de 2011 | Revisado: enero 22 de 2012 | Aceptado: febrero 26 de 2012

\author{
Mónica Bernaldo-De-Quirós ARagón ** \\ FRANCISCO J. LABRADOR ENCINAS \\ Francisco J. ESTUPiñá PUIG \\ IGNACIO FERNÁNDEZ-ARIAS \\ Universidad Complutense de Madrid, España
}

\section{RES U MEN}

El objetivo de este trabajo fue analizar las diferencias entre los casos de corta (5-10 sesiones), media (10-18 sesiones) y larga duración (más de 18 sesiones) en variables sociodemográficas, clínicas y de tratamiento. Se analizaron los datos de 349 pacientes de la Clínica Universitaria de Psicología de la Universidad Complutense de Madrid (CUP-UCM), que habían terminado el tratamiento con éxito. El número de técnicas aplicadas durante la intervención fue la variable más discriminativa, seguida por la comorbilidad -haber recibido un tratamiento anterior- número de tratamientos anteriores y número de objetivos establecidos antes de la intervención. Los resultados señalan la importancia de identificar las técnicas más eficaces para cada problema u objetivo para reducir, en la medida de lo posible, la duración de las intervenciones sin disminuir su eficacia.

Palabras clave autores

Tratamientos empíricamente apoyados, duración de tratamientos, comorbilidad.

Palabras clave descriptores

Psicología Clínica, Intervención, psicoterapia.

\section{A B S T R A C T}

The aim of this study was to analyze differences among cases of short (5-10 sessions), medium (11-18 sessions) and long term (more than 18 sessions) in sociodemografic, clinical and treatment variables. Data from 349 patients of the Clínica Universitaria de Psicología de la Universidad Complutense de Madrid (CUP-UCM), who had successfully completed treatment, were analyzed. Number of intervention techniques was the most significant variable, followed by comorbidity, previous treatment, number of previous treatments and number of therapeutic objectives. The results indicate the importance of identifying which techniques are more effective for each problem or objective in order to reduce, as far as possible, the extension of the treatment without decreasing its effectivity.

Key words authors

Empirically supported treatments, duration of treatment, comorbidity.

Key words plus

Clinical Psychology, Intervention, Psychotherapy. 


\section{Introducción}

Uno de los retos importantes a los que se enfrenta la Psicología Clínica actual es conseguir resultados positivos, y además hacerlo en un breve período de tiempo. Para esto se han desarrollado protocolos de tratamiento para los diferentes tipos de trastornos. Estos protocolos, en algunos casos, han obtenido el adecuado apoyo empírico que avala la consecución de éxito terapéutico, tanto en el postratamiento como en los seguimientos, en un reducido número de sesiones (Bados, 2005; Labrador \& Alonso, 2007; Lejuez, Hopko \& Hopko, 2001; Mueser et al., 2008; Öst, Alm, Brandberg \& Breitholtz, 2001).

Por otro lado, múltiples trabajos destacan la importancia de conseguir mejorías en las primeras sesiones, señalando que conforme se prolongan los tratamientos estas se van reduciendo. Así, Howard, Kopta, Krause y Or-linsky (1986) señalan que el $58 \%$ de los pacientes mejoran en las primeras siete sesiones y que, a partir de la vigésimo sexta, el número de pacientes que obtienen mejoría sigue una curva negativamente acelerada. En la misma línea, Shapiro, Rees, Barkham y Hardy (1995) encuentran que ocho sesiones producen los mismos logros terapéuticos que dieciséis en el tratamiento para la depresión. Resultados similares apuntan Baldwin, Berkeljon, Atkins, Olsen y Nielsen (2009); Barkham et al. (2006) y Kopta (2003), indicando que un número elevado de sesiones de tratamiento comienzan bien a "intoxicar" la eficacia de la intervención, bien a mantener estáticas las mejorías que parecen seguir una evolución asintótica.

El hecho de que un mayor número de sesiones no siempre esté acompañado de un incremento en la mejoría resulta extraño y lleva a cuestionar por qué en algunos casos se prolongan, incluso de forma significativa, el número de sesiones de tratamiento.

Algunos trabajos analizan la influencia de factores no clínicos, al respecto. Así, Barnow, Linden y Schaub (1997) encuentran que ser mujer, mayor de 46 años y estar viuda o divorciada se relacionan con una prolongación de la hospitalización en el tratamiento de la depresión. Por el contrario, Koss (1980) señaló que variables como sexo, ocupación, estado civil, edad, educación y cociente intelectual no influían en un mayor número de sesiones.

Tradicionalmente, se atribuye una mayor duración del tratamiento a ciertos grupos diagnósticos como la esquizofrenia, los trastornos por consumo de sustancias o de la conducta alimentaria. En esta dirección apuntan algunos trabajos que señalan la importancia de trabajar múltiples objetivos terapéuticos y utilizar programas multicomponentes más amplios, con el fin de prevenir recaídas en estos trastornos (Saldaña, 2001; Secades \& Fernández, 2001; Vallina \& Lemos, 2001), lo que requiere tratamientos más prolongados.

La comorbilidad es otra de las variables más mencionadas. En los estudios de eficacia se excluye a los pacientes que presentan otro tipo de patologías, pero en la práctica clínica habitual se supone que la comorbilidad es algo habitual. Diversos trabajos señalan que la presencia de esta variable lleva a peores resultados y a la prolongación de los tratamientos (Deveney \& Otto, 2010; Morrison, Bradley \& Westen, 2003; O’Connor \& Stewart, 2010; Quiroga \& Errasti, 2001).

Para Lin (1998) haber recibido tratamientos psicológicos previos es otro de los factores que generan terapias más largas. Muñiz (2004) indica que la duración del tratamiento está relacionada con la existencia de algún tratamiento anterior, el uso de medicación y la gravedad y tiempo de evolución de la queja. Sin embargo, Koss (1979) no encuentra relación entre haber recibido tratamientos psicológicos previamente y la duración del tratamiento.

En un estudio previo (Labrador, Bernaldo de Quirós \& Estupiñá, 2011) se encontró que los factores que mejor explicaban la prolongación del tratamiento eran, en este orden, el número de técnicas aplicadas durante la intervención, la presencia de comorbilidad y el hecho de haber recibido un tratamiento anterior. Sin embargo, estos resultados estaban limitados por el bajo porcentaje de varianza explicado, en gran medida, por la variabilidad intersujetos. Esto llevó a analizar los casos extremos de corta (5 sesiones o menos) y larga duración (20 sesiones o más), encontrando diferencias significativas en la cantidad de técnicas aplicadas durante la intervención, el número de objetivos planteados 
al comienzo de la intervención, el grupo diagnóstico, comorbilidad, el hecho de haber recibido algún tratamiento anterior, la edad y el nivel educativo. No obstante, el número de sesiones recibidas por los casos de corta duración era muy bajo $(\leq 5)$ lo que podría limitar también la interpretación de los resultados.

El objetivo del presente estudio fue analizar si existen diferencias en variables sociodemográficas, clínicas y de tratamiento, entre los casos que han obtenido el alta terapéutica, según la duración del tratamiento recibido (corta, media y larga).

\section{Método}

\section{Participantes}

Los participantes fueron 349 pacientes que demandaron ayuda psicológica en la Clínica Universitaria de Psicología de la Universidad Com- plutense de Madrid (CUP-UCM), que habían recibido al menos cinco sesiones de tratamiento y que habían finalizado con éxito la terapia. Las características de la clínica y de los terapeutas están descritos en otro trabajo (Labrador, Estupiñá \& García-Vera, 2010).

Como puede observarse en la Tabla 1, en la muestra total $(N=349)$, la mayoría de los pacientes son mujeres $(70.2 \%)$, solteras $(66.8 \%)$, aproximadamente la mitad estudiantes (45.3\%) o trabajadoras $(52.6 \%)$ y con al menos estudios secundarios, (83.6\%).

Como señala la Tabla 2, en la muestra total $(\mathrm{N}=349)$ destaca que el principal grupo diagnóstico es trastornos ansiedad (37\%), el 33.5\% presenta diagnóstico comórbido, la mitad (51\%) había recibido un tratamiento anterior, la mayoría no recibió apoyo farmacológico (79.1\%). Se estableció una media de 7.7 objetivos terapéuticos y se aplicó una media de 7.2 técnicas de intervención.

\section{TABLA 1}

Características sociodemográficas

\begin{tabular}{|c|c|c|c|c|}
\hline & $\begin{array}{c}\text { Casos de corta } \\
\text { duración } \\
N=120\end{array}$ & $\begin{array}{c}\text { Casos de media } \\
\text { duración } \\
N=125\end{array}$ & $\begin{array}{c}\text { Casos de larga } \\
\text { duración } \\
N=104\end{array}$ & $\begin{array}{c}\text { Total de la } \\
\text { muestra } \\
N=349\end{array}$ \\
\hline $\begin{array}{l}\text { Edad M (DE) } \\
\text { (Rango) }\end{array}$ & $\begin{array}{c}27.9(12.5) \\
(4-64)\end{array}$ & $\begin{array}{c}31.5(12.9) \\
(6-68)\end{array}$ & $\begin{array}{c}29.8(12.2) \\
(5-58)\end{array}$ & $\begin{array}{c}29.7(12.6) \\
\quad(4-68)\end{array}$ \\
\hline $\begin{array}{l}\text { Sexo }(\%) \\
\text { Varones } \\
\text { Mujeres } \\
\end{array}$ & $\begin{array}{l}26.7 \\
73.3 \\
\end{array}$ & $\begin{array}{l}28.8 \\
71.2 \\
\end{array}$ & $\begin{array}{l}34.6 \\
65.4 \\
\end{array}$ & $\begin{array}{l}29.8 \\
70.2 \\
\end{array}$ \\
\hline $\begin{array}{l}\text { Estado Civil (\%) } \\
\text { Solteros } \\
\text { Casados } \\
\text { Separados/Divorciados/Viudos }\end{array}$ & $\begin{array}{r}70 \\
24.2 \\
5.8 \\
\end{array}$ & $\begin{array}{r}60 \\
34.4 \\
5.6\end{array}$ & $\begin{array}{r}71.2 \\
26.9 \\
1.9 \\
\end{array}$ & $\begin{array}{c}66.8 \\
28.7 \\
4.6 \\
\end{array}$ \\
\hline $\begin{array}{l}\text { Nivel de estudios (\%) } \\
\text { Primarios incompletos } \\
\text { Primarios completos } \\
\text { Secundarios } \\
\text { Diplomados Universitarios } \\
\text { Licenciados }\end{array}$ & $\begin{array}{r}8.3 \\
9.2 \\
24.2 \\
23.3 \\
35\end{array}$ & $\begin{array}{c}6.4 \\
12 \\
30.4 \\
18.4 \\
32.8 \\
\end{array}$ & $\begin{array}{r}4.8 \\
7.7 \\
35.6 \\
20.2 \\
31.7 \\
\end{array}$ & $\begin{array}{r}6.6 \\
9.7 \\
29.8 \\
20.6 \\
33.2 \\
\end{array}$ \\
\hline $\begin{array}{l}\text { Profesión /Situación laboral (\%) } \\
\text { Estudiante } \\
\text { Profesional/Técnico } \\
\text { Personal Administración y Servicios } \\
\text { Ama de casa } \\
\text { Directivo/Gerente/ Empresario } \\
\text { Obrero } \\
\text { Parados/Jubilados } \\
\text { Otros }\end{array}$ & $\begin{array}{c}45.8 \\
17.5 \\
19.1 \\
2.5 \\
5 \\
1.6 \\
4.2 \\
4.2 \\
\end{array}$ & $\begin{array}{c}43.2 \\
9.6 \\
19.2 \\
4 \\
7.2 \\
3.2 \\
4 \\
8 \\
\end{array}$ & $\begin{array}{c}47.1 \\
18.3 \\
13.5 \\
3.8 \\
2 \\
4.8 \\
5.8 \\
4.8\end{array}$ & $\begin{array}{r}45.3 \\
17.5 \\
14.9 \\
3.4 \\
4.9 \\
3.2 \\
4.6 \\
5.7 \\
\end{array}$ \\
\hline
\end{tabular}

Fuente: elaboración propia. 
TABLA 2

Características clínicas y de tratamiento

\begin{tabular}{|c|c|c|c|c|}
\hline & $\begin{array}{c}\text { Casos de corta } \\
\text { duración } \\
N=120\end{array}$ & $\begin{array}{l}\text { Casos de media } \\
\text { duración } \\
N=125\end{array}$ & $\begin{array}{c}\text { Casos de larga } \\
\text { duración } \\
N=104\end{array}$ & $\begin{array}{c}\text { Total de la } \\
\text { muestra } \\
N=349\end{array}$ \\
\hline $\begin{array}{l}\text { Grupo diagnóstico (\%) } \\
\text { T por consumo de sustancias } \\
\text { Esquizofrenia } \\
\text { T del estado del ánimo } \\
\text { T de ansiedad } \\
\text { T adaptativos } \\
\text { T disociativos } \\
\text { T somatomorfos } \\
\text { T de la conducta alimentaria } \\
\text { T sexuales } \\
\text { T del control de impulsos } \\
\text { T de la personalidad } \\
\text { T inicio en infancia, niñez o adolescencia } \\
\text { Otros problemas objeto de atención clínica } \\
\text { T del sueño } \\
\text { Códigos adicionales o sin diagnóstico } \\
\end{array}$ & $\begin{array}{c}0 \\
1.7 \\
7.5 \\
35 \\
8.3 \\
0 \\
0.8 \\
0.8 \\
2.5 \\
2.5 \\
2.5 \\
5 \\
20 \\
0.8 \\
12.5\end{array}$ & $\begin{array}{c}0.8 \\
2.4 \\
11.2 \\
41.6 \\
6.4 \\
2.4 \\
1.6 \\
0 \\
0.8 \\
2.4 \\
4.8 \\
4.8 \\
11.2 \\
0.8 \\
8.8\end{array}$ & $\begin{array}{c}1 \\
2.9 \\
11.5 \\
34.6 \\
3.8 \\
0 \\
3.8 \\
4.8 \\
1.9 \\
2.9 \\
6.7 \\
2.9 \\
13.5 \\
0 \\
9.6\end{array}$ & $\begin{array}{c}0.6 \\
2.3 \\
10 \\
37.2 \\
6.3 \\
0.9 \\
2 \\
1.7 \\
1.7 \\
2.6 \\
4.6 \\
4.3 \\
14.9 \\
0.6 \\
10.3\end{array}$ \\
\hline $\begin{array}{l}\text { Comorbilidad (\%) ** } \\
\text { No } \\
\text { Sí }\end{array}$ & $\begin{array}{l}76.7 \\
23.3\end{array}$ & $\begin{array}{l}64 \\
36 \\
\end{array}$ & $\begin{array}{l}57.7 \\
42.3\end{array}$ & $\begin{array}{l}66.5 \\
33.5\end{array}$ \\
\hline $\begin{array}{l}\text { Tratamientos anteriores (\%) ** } \\
\text { No } \\
\text { Sí }\end{array}$ & $\begin{array}{l}59.2 \\
40.8\end{array}$ & $\begin{array}{l}48 \\
52\end{array}$ & $\begin{array}{l}38.5 \\
61.5\end{array}$ & $\begin{array}{l}49 \\
51\end{array}$ \\
\hline $\begin{array}{l}\text { № tratamientos anteriores* } \\
\text { M (DE) (Rango) }\end{array}$ & $\begin{array}{c}0.56(0.8) \\
(0-4)\end{array}$ & $\begin{array}{c}0.8(0.9) \\
(0-5)\end{array}$ & $\begin{array}{l}0.9(0.9) \\
(0-4)\end{array}$ & $\begin{array}{l}0.7(0.9) \\
(0-5)\end{array}$ \\
\hline $\begin{array}{l}\text { № objetivos* } \\
\text { M (DT) (Rango) }\end{array}$ & $\begin{array}{c}6.8(3.2) \\
(1-20)\end{array}$ & $\begin{array}{l}8.2(3.3) \\
(2-19)\end{array}$ & $\begin{array}{c}8.5(5.1) \\
(1-30)\end{array}$ & $\begin{array}{c}7.7(3.9) \\
(1-30)\end{array}$ \\
\hline $\begin{array}{l}\text { № técnicas **** } \\
\text { M (DT) (Rango) }\end{array}$ & $\begin{array}{c}6.6(2.2) \\
(1-12)\end{array}$ & $\begin{array}{l}7.5(2.1) \\
(2-13)\end{array}$ & $\begin{array}{c}7.7(1.8) \\
(3-12)\end{array}$ & $\begin{array}{c}7.2(2.1) \\
(1-13)\end{array}$ \\
\hline $\begin{array}{l}\text { Prescripción de psicofármacos (\%) } \\
\text { No } \\
\text { Sí }\end{array}$ & $\begin{array}{l}85.8 \\
14.2\end{array}$ & $\begin{array}{l}74.4 \\
25.6\end{array}$ & $\begin{array}{l}76.9 \\
23.1\end{array}$ & $\begin{array}{l}79.1 \\
20.9\end{array}$ \\
\hline
\end{tabular}

$* p<0.05 ; * * p<0.01 ; * * *<0.001$.

Fuente: elaboración propia.

\section{Variables}

Se tomaron como dependientes diversas variables sociodemográficas, clínicas y de tratamiento.

\section{Sociodemográficas}

Mediante un cuestionario construido ad hoc se recogía el sexo, la edad, el estado civil, la profesión, la situación laboral y el nivel de estudios.

\section{Características clínicas}

Recogidas en el proceso de evaluación mediante entrevista clínica, registros y cuestionarios: grupo diagnóstico al que pertenece el dictamen establecido por los terapeutas (según criterios DSMIV-TR), comorbilidad (presencia de un segundo diagnóstico), prescripción de fármacos, número de tratamientos anteriores. 


\section{Características del tratamiento}

Obtenidas a partir del informe de resultados de la intervención: número de objetivos terapéuticos establecidos al comienzo del tratamiento y cantidad de técnicas aplicadas durante la intervención.

Se utilizó como variable independiente la duración del tratamiento, estableciéndose tres grupos (corta, media y larga duración), en función del número de sesiones.

\section{Procedimiento}

De la muestra total de casos de la CUP-UCM que habían acudido a tratamiento entre 1999 y 2010, se seleccionó a todos los pacientes que habían recibido al menos cinco sesiones de tratamiento y cuyo tratamiento ya había finalizado con éxito (altas), es decir, se habían conseguido todos los objetivos terapéuticos propuestos al comienzo de la intervención. Se obtuvo una muestra de 349 pacientes.

Para establecer los grupos con un número similar de pacientes, se utilizaron los percentiles referidos al número de sesiones; así se consideraron:

a) Casos de corta duración, aquellos que habían recibido entre 5 y 10 sesiones de tratamiento $(N=120)$

b) Casos de media duración, todos aquellos que habían recibido entre 11 y 18 sesiones $(N=125)$;

c) Casos de larga duración, aquellos que habían recibido más de 18 sesiones de tratamiento $(N=104)$.

\section{Análisis de datos}

Se realizaron análisis descriptivos y de frecuencias para identificar las características sociodemográficas, clínicas y de tratamiento de la muestra total y por grupos.

Las diferencias entre los tres grupos en las variables cualitativas (sexo, estado civil, nivel educativo, situación laboral, comorbilidad, haber recibido un tratamiento anterior) se contrastaron mediante la prueba de chi cuadrado de Pearson.

Para comprobar si existían diferencias entre los tres grupos en las variables cuantitativas (edad, número de tratamientos anteriores, número de objetivos planteados antes de la intervención, número de técnicas aplicadas), se realizaron análisis de varianza. Se comprobó la homogeneidad de las varianzas con la prueba de Levene, en los casos en los que la varianza de los grupos no era igual; en lugar de ANOVA, se utilizó la prueba robusta de igualdad de medias de Welch para comprobar si existían diferencias significativas entre las medias. Se realizó un análisis posterior (prueba a posteriori de Tukey) para comprobar la diferencia entre todos los pares de las medias.

\section{Resultados}

\section{Diferencias en variables sociodemográficas}

La Tabla 1 muestra las características sociodemográficas de los casos de corta, media y larga duración. No se observó ninguna diferencia significativa entre los tres grupos en las variables sociodemográficas.

\section{Diferencias en variables \\ clínicas y de tratamiento}

La Tabla 2 muestra las características clínicas y de tratamiento de los casos de corta, media y larga duración, así como sus diferencias significativas.

Se observaron diferencias significativas en comorbilidad $\left(\chi_{2}^{2}=9.5 ; p<0.01\right)$; los casos de corta duración presentaban una comorbilidad del $23.3 \%$ mientras que en los casos de larga era del $42.3 \%$. También aparecieron diferencias significativas en función de haber recibido un tratamiento anterior $\left(\chi_{2}^{2}=9.6 ; p<0.01\right)$. La mayoría de los casos de larga duración (61.5\%) había recibido un tratamiento anterior, frente a una minoría (40.8\%) de los casos de corta duración. Asimismo, fueron significativas las diferencias en el número de tratamientos anteriores $\left(F_{(2,346)}=3.91 ; p<0.05\right)$ entre los casos de corta y larga duración.

Dado que en el número de objetivos terapéuticos al comienzo de la intervención la varianza de los grupos no era igual $\left(F_{(2,208)}=3.59 ; p<0.05\right)$, se utilizó la tabla de Welch, que confirmó la existencia 
de diferencias significativas en el número de objetivos $\left(F_{(2,121,53)}=4.36 ; p<0.05\right)$ entre los casos de corta y larga duración.

También aparecieron diferencias significativas en el número de técnicas aplicadas durante la intervención $\left(F_{(2,346)}=8.83 ; p<0.001\right)$ entre los casos de corta y media duración $(p<0.01)$, y corta y larga duración $(p<0.001)$.

Sin embargo, no se observaron diferencias significativas en grupo diagnóstico $\left(\chi_{2}^{2}=4.9 ; p=0.08\right)$, ni en la en la prescripción de fármacos $\left(\chi_{2}^{2}=5.2 ; p\right.$ $=0.07)$ entre los distintos grupos, aunque los valores estaban próximos a la significación.

\section{Discusión}

El paciente tipo que acude en busca de ayuda psicológica presenta un perfil sociodemográfico -confirmando lo señalado en otros trabajos- caracterizado por ser mujer, soltera, de unos 30 años, empleada o estudiante, y al menos un 50\% con estudios universitarios (Labrador et al., 2010; Vallejo et al., 2008). En cuanto a características clínicas, predominan los trastornos de ansiedad, sin diagnóstico comórbido, con un tratamiento anterior (51\%) y sin ingesta de fármacos (79.1\%).

No se encontraron diferencias significativas en las variables sociodemográficas entre los casos de corta, media y larga duración. Todos los grupos presentan el mismo perfil del promedio de la muestra total. Tal y como señalan otros estudios (Estupiñá et al., 2008; Koss, 1980), las variables sociodemográficas no parecen variables responsables de la prolongación de tratamientos. Las diferencias aparecidas en un estudio anterior (Labrador et al., 2011) hay que tomarlas con precaución, dado que se seleccionó como casos de corta duración a los pacientes que habían tenido un número menor a cinco sesiones. No obstante, conviene llamar la atención sobre el aumento de los porcentajes de hombres conforme se incrementa la duración del tratamiento (26\%, 28\%, 34.6\%); también, al reducido porcentaje de pacientes con tratamiento farmacológico.

Como variables clínicas más relevantes en la duración del tratamiento aparecen la presencia de comorbilidad y los tratamientos anteriores. Los casos de larga duración presentaban un porcentaje de comorbilidad (42.3\%) significativamente mayor que los casos de corta duración (23.3\%). Estos resultados coinciden con los de otros estudios (Deveney \& Otto, 2010; Labrador et al., 2011; Morrison et al., 2003). Siempre se ha señalado que la comorbilidad es una de las variables que más diferencia la investigación de la práctica clínica, ya que en la primera se excluye habitualmente a los pacientes con comorbilidad, cosa que no sucede en la segunda. Los porcentajes de comorbilidad de este estudio (33.5\%) son cercanos a los encontrados habitualmente, entre el 30 y 45\% (Kessler, Chiu, Demler \& Walters, 2005; Pini, Perkonnig, Tansella \& Wittchen, 1999; Roca et al., 2009). Resulta evidente que la comorbilidad suele conllevar un mayor número de objetivos terapéuticos. Los datos aquí obtenidos confirman empíricamente lo esperado: la comorbilidad es un factor de especial relevancia asociado a la prolongación de los tratamientos.

También el hecho de haber recibido un tratamiento previo se relaciona con la prolongación en el tratamiento actual. En los casos de larga duración, el 61.5\% había recibido un tratamiento previo, frente al $40.8 \%$ de los casos de corta duración. Lo mismo que el número de tratamientos previos. Probablemente, la presencia de uno o más tratamientos previos, lógicamente fracasados, sea un índice de la gravedad o cronicidad del problema, factores ambos que harían más laboriosa la intervención prolongándola.

Estos resultados apoyan los obtenidos en la mayor parte de los trabajos anteriores (Lin, 1998; Muñiz, 2004), también los obtenidos por nuestro grupo (Labrador et al., 2011), aunque algunos trabajos esporádicos, como el de Koss (1979), no encontraran esta relación. Es de esperar que una historia previa de fracasos terapéuticos sea indicativo de mayores dificultades, bien por las características del problema, bien por las características del paciente y su entorno, a lo que deberá responder el terapeuta con un trabajo especialmente minucioso en la evaluación para detectar las causas del fracaso previo y obrar en consecuencia.

El que no se confirme la hipótesis de diferencias en la duración del tratamiento según diagnósticos, 
aunque incuestionable con los datos obtenidos, puede deberse a la muestra utilizada en el trabajo. La literatura indica que ciertos diagnósticos como esquizofrenia, trastornos de la conducta alimentaria o trastornos por consumo de sustancias requieren un mayor número de sesiones de tratamiento (Saldaña, 2001; Secades \& Fernández, 2001; Vallina \& Lemos, 2001). No obstante, también es cierto que no son los casos que con más frecuencia acuden a una clínica de psicología, sino que tales pacientes suelen acudir a recursos especializados. De hecho, en la muestra del presente estudio es mínimo el número de casos de esquizofrenia $(N=8)$, TCA $(N=6)$ y abuso de sustancias $(N=2)$, lo que hace que deba relativizarse este resultado de no diferencias.

Sin duda, la variable más relevante es el número de técnicas aplicadas durante la intervención, los casos de corta duración recibieron un número de técnicas significativamente menor que los casos de media y larga duración, confirmando los resultados del estudio anterior (Labrador et al., 2011). Parece lógico que cuantas más técnicas se apliquen mayor sea el número de sesiones, pero por otro lado el incremento en número de sesiones no mejora en muchos casos la eficacia de la intervención (Baldwin et al., 2009; Howard et al., 1986; Kopta, 2003; Olivares, Sánchez-Meca \& Rosa, 1999; Shapiro et al., 1995). En consecuencia, un reto importante para reducir la duración del tratamiento debe pasar por disminuir el número de técnicas aplicadas, eso sí, sin disminuir la eficacia de la intervención. Se revela por lo tanto fundamental averiguar cuáles son las técnicas realmente eficaces para cada problema u objetivo, y si algunas técnicas se usan más para "tranquilidad" del terapeuta que por necesidades estrictas del caso.

También el número de objetivos planteados al comienzo de la intervención es significativamente mayor en los casos de larga duración que en los de corta duración, confirmando asimismo los resultados del estudio anterior (Labrador et al., 2011). Señalar las técnicas más efectivas y eficientes para lograr cada objetivo terapéutico, o incluso para cada conducta, puede ser un logro más práctico incluso que el de establecer el tratamiento más adecuado para cada categoría diagnóstica. Considerar más específicamente los objetivos de cambio permitirá una valoración más precisa de la función que tienen las conductas problema para el paciente y orientará mejor la elección de tratamiento.

Probablemente, si los psicólogos precisaran mejor los objetivos terapéuticos las intervenciones serían más precisas, breves y eficaces. En esta dirección sería importante desarrollar protocolos de intervención específicos por objetivos, no por diagnósticos, indicando las técnicas más adecuadas para lograrlo. No se trata de conseguir personas perfectas (iexisten?), sino de ayudar a los pacientes a solucionar sus problemas, a mejorar su calidad de vida, considerando que los problemas son conductas no diagnósticos.

No obstante, este estudio no está exento de limitaciones derivadas del tipo de clínica en la que se realiza el estudio, que si bien sus características son similares a las de una clínica privada (Labrador et al., 2010), el porcentaje de estudiantes universitarios es elevado.

\section{Referencias}

Bados, A. (2005). Fobias especificas. Barcelona: Universidad de Barcelona.

Baldwin, S. A., Berkeljon, A., Atkins, D. C., Olsen, J. A. \& Nielsen, S. L. (2009). Rates of change in naturalistic psychotherapy: Contrasting dose-effect and good-enough level models of change. Journal of Consulting and Clinical Psychology, 77(2), 203-211.

Barkham, M., Connell, J., Miles, J. N., Evans, C., Stiles, W. D., Margison, F., et al. (2006). Dose-effects relations and responsive regulation of treatment duration: The good-enough level. Journal of Consulting and Clinical Psychology, 74(1), 160-167.

Barnow, S., Linden, M. \& Schaub, R. T. (1997). The impact of psychosocial and clinical variables on duration of inpatient treatment for depression. Social Psychiatry and Psychiatric Epidemiology, 32(6), 312-316.

Deveney, C. M. \& Otto, M. W. (2010). Resolving treatment complications associated with comorbid depression. En M. W. Otto \& S. G. Hofmann (Eds.), Avoiding treatment failures in the anxiety disorders (pp. 231-249). New York: Springer Sciences. 
Estupiñá, F. J., Aranda, I., Ballesteros, F., Sánchez, E. M., García-Vera, M. P. \& Sanz, J. (2008, noviembre). iExiste un perfil de pacientes que prediga una prolongación del tratamiento de la depresión? Póster presentado en la IV Reunión Anual de la SEPCyS, Zaragoza, España.

Howard, I. K., Kopta, S. M., Krause, M. S. \& Or-linsky, D. E. (1986). The dose-effect relationship in psychotherapy. American Psychologist, 41(2), 159-164.

Kessler, R. C., Chiu, W. T., Demler, O. \& Walters, E. E. (2005). Prevalence, severity, and comorbidity of 12-month DSM-IV disorders in the national comorbidity survey replication. Archives of General Psychiatry, 62(6), 617-627.

Kopta, S. (2003). The dose-effect relationship in psychotherapy: A defining achievement for Dr. Kenneth Howard. Journal of Clinical Psychology, 59(7), 727-733.

Koss, M. P. (1979). Length and psychotherapy for clients seen in private practice. Journal of Consulting and Clinical Psychology, 47(1), 210-212.

Koss, M. P. (1980). Descriptive characteristics and length of psychotherapy of child and adult clients seen in private psychological practice. Psychotherapy: Theory, Research \& Practice, 17(3), 268-271.

Labrador, F. J. \& Alonso, E. (2007). Evaluación a corto plazo de un programa de intervención para el trastorno de estrés postraumático en mujeres mexicanas víctimas de violencia doméstica. Revista de Psicopatología y Psicología Clínica, 12(2), 117-130.

Labrador, F. J., Estupíná, F. J. \& García-Vera, M. P. (2010). Demanda de atención psicológica en la práctica clínica. Psicothema, 22(4), 619-626.

Labrador, F. J., Bernaldo de Quirós, M. \& Estupiñá, F. J. (2011). ¿Por qué se alargan los tratamientos psicológicos?: predictores de una mayor duración de tratamiento y diferencias entre los casos de corta y larga duración. Psicothema, 23(4), 573-579.

Lejuez, C. W., Hopko, D. R. \& Hopko, S. D. (2001). A brief behavioral activation treatment for depression. Treatment manual. Behavior Modification, 25(2), 255-286.

Lin, J. C. H. (1998). Descriptive characteristics and length of psychotherapy of Chinese American clients seen in private practice. Professional Psychology: Research and Practice, 29(6), 571-573.
Morrison, K., Bradley, R. \& Westen, D. (2003). The external validity of controlled clinical trials of psychotherapy for depression and anxiety: A naturalistic study. Psychology and Psychotherapy, 76(2), 109-132.

Mueser, K. T., Rosenberg, S. D., Xie, H., Jankowski, M. K., Bolton, E., Lu, W., et al. (2008). A randomized controlled trial of cognitive-behavioral treatment for posttraumatic stress disorder in severe mental illness. Journal of Consulting and Clinical Psychology, 76(2), 219-271.

Muñiz, J. A. (2004). Informe sobre una evaluación de resultados del modelo de terapia familiar breve en el ámbito privado. Papeles del Psicólogo, 87, 29-34.

Olivares, J., Sánchez-Meca, J. \& Rosa, A. I. (1999). Eficacia de las intervenciones conductuales en los problemas de ansiedad en España. Psicología Conductual, 7(2), 283-300.

Öst, L., Alm, T., Brandberg, M. \& Breitholtz, E. (2001). One vs. five sessions of exposure and five sessions of cognitive therapy in the treatment of claustrophofia. Behavior Research and Therapy, 39(2), 167-183.

O’Connor, R. M. \& Stewart, S. H. (2010). Substance use disorders. En D. Mckay, J. S. Abramowitz \& S. Taylor (Eds.), Cognitive-behavioral therapy for refractory cases: Turning failure into success (pp. 211-229). Washington, DC: American Psychological Association.

Pini, S., Perkonnig, A., Tansella, M. \& Wittchen, H. U. (1999). Prevalence and 12-month outcome of subthreshold mental disorders in primary care. Journal of Affective Disorders, 56(1), 37-48.

Quiroga, R. \& Errasti, J. M. (2001). Tratamientos psicológicos eficaces para los trastornos de personalidad. Psicothema, 13(3), 393-406.

Roca, M., Gili, M., García-García, M., Salva, J., Vives, M., García Campayo, J., et al. (2009). Prevalence and comorbidity of common mental disorders in primary care. Journal of Affective Disorders, 119(1), 52-58.

Saldaña, C. (2001). Tratamientos psicológicos eficaces para trastornos del comportamiento alimentario. Psicothema, 13(3), 381-392.

Secades, R. \& Fernández, J. R. (2001). Tratamientos psicológicos eficaces para la drogadicción: nicoti- 
na, alcohol, cocaína y heroína. Psicothema, 13(3), 365-380.

Shapiro, D., Rees, A., Barkham, M. \& Hardy, G. (1995). Effects of treatment duration and severity of depression on the maintenance of gains after cognitive-behavioural and psychodynamic interpersonal psychotherapy. Journal of Consulting and Clinical Psychology, 63(3), 378-387.
Vallejo, M. A., Jordán, C. M., Díaz, M. I., Comeche, M. I., Ortega, J., Amor, P., et al. (2008). Perfil sociodemográfico de los usuarios de un servicio de asistencia psicológica online. Psicothema, 20(4), 610-615.

Vallina, O. \& Lemos, S. (2001). Tratamientos psicológicos eficaces para la esquizofrenia. Psicothema, 13(3), 345-364. 
